\title{
Alcohol and Narcolepsy: A Case Report
}

\author{
Richa Tripathi ${ }^{1}$, Ahmed Osama Javed $^{2}$
}

\begin{abstract}
Keywords: Alcohol, Insomnia, Narcolepsy, Sleep disorders.

Indian Journal of Sleep Medicine (2021): 10.5005/jp-journals-10069-0069
\end{abstract}

Alcohol has a varied effect on sleep. It affects daytime alertness and also certain physiological processes during sleep. It affects various parts of the central nervous system including the reticular activating system. Many other sleep disorders besides insomnia have been observed in alcohol-dependent patients. The present article discusses about two cases of narcolepsy caused by alcohol use.

\section{Statement of Significance}

Alcohol causes various sleep disorders with varied presentations. Sleep disorders should be a part of the routine evaluation in substance use disorder patients and can be comorbid with other disorders as well.

\section{INTRODUCTION}

Alcohol affects sleep, daytime alertness, and certain physiological processes that occur during sleep. Alcohol presents sedative or stimulant effects depending on dosage and individual susceptibility. ${ }^{1}$ Lower doses may increase total sleep time, whereas higher doses may lead to short-term withdrawal, increasing sympathetic activity, and sleep disruption especially during the second half of the night. Alcohol also exacerbates sleep-related breathing disorder.

Alcohol causes other sleep disorders such as narcolepsy and hypersomnia which are also reported in the literature. In a case-control study conducted by Barateau et al., ${ }^{2}$ narcolepsies, both type 1 (NT1) and type 2 (NT2), were observed with the use of various substances.

The present case series will discuss two such patients who were suffering from narcolepsy due to their chronic alcohol use.

\section{Case Descriptions}

\section{Case 1}

The patient was a 45-year-old married male who presented to us with symptoms of low mood, lack of interest, and falling down from bed while asleep. He worked in a private firm. Fifteen years back he began to consume alcohol along with friends which gradually increased to 250-500 mL of IMFL (1.5-2.5 quarters) daily in dependent pattern. He would be often intoxicated and typically slept only 3-4 hours before waking to start work the following day. He subsequently developed acute daytime drowsiness for periods of 10 minutes at a time while driving or walking. Gradually his daytime drowsiness increased and he would sleep anywhere if sitting for 10 minutes or more. He also started experiencing episodes of sudden loss of motor power while fully conscious (cataplexy) that lasted for a few seconds at a time. He also had nighttime insomnia and nightmares. The symptoms were initially relatively mild, but 4 years back he had fallen down while sleeping on a berth in a railway coach and injured himself. Over the next 3 years, the symptoms gradually got worse; his daytime drowsiness increased much and he would fall daily
1,2Department of Psychiatry, All India Institute of Medical Sciences, Gorakhpur, Uttar Pradesh, India

Corresponding Author: Richa Tripathi, Department of Psychiatry, All India Institute of Medical Sciences, Gorakhpur, Uttar Pradesh, India, Phone:+91 09999457412, e-mail: drricha12@gmail.com

How to cite this article: Tripathi R, Javed AO. Alcohol and Narcolepsy: A Case Report. J Sleep Med 2021;16(2):59-60.

Source of support: Nil

Conflict of interest: None

from bed while sleeping. The patient had reduced his alcohol intake for last 3 years and claimed to be abstinent for last 1 month at the time of presentation. He had consulted previously in the medicine department but did not report any improvement in his condition. There was no history of complicated alcohol withdrawal and features of liver dysfunction. He did not report symptoms of any other medical disorder or have any surgery in past. His general physical examination revealed no abnormality and in mental status examination his affect was depressed, and he had ideas of hopelessness and helplessness, his higher mental functions were intact.

\section{Management and Outcome}

The patient was evaluated on the Epworth sleepiness scale for his daytime drowsiness and had a score of 15 (moderate excessive daytime sleepiness). Blood investigations-CBC, RFT, LFT, Vit B12, D3, thyroid profile, blood sugar, ECG, and MRI brain-were done. His vitamin B12 level was low and other blood parameters were normal. His scans were also normal. A diagnosis of alcohol-induced narcolepsy was made and he was started on tab sertraline, tab modafinil, and tab clonazepam. Motivational enhancement therapy and relapse prevention therapy was started for his alcohol use. He was also psychoeducated regarding illness and need to be abstinent. The patient reported improvement in his symptoms after 2 weeks.

\section{Case 2}

A 67-year-old widower male presented to us with excessive sleepiness during the daytime for the last 20 years. Previously it was while sitting idle, reading, or watching television for 15 to 20 minutes. For the last 5 years, it had increased and he would feel sleepy while driving also. He would have to pause his driving, drink 
some water to make himself aroused and active. He also reported snoring during the night which was reported to him by family members. However, he did not report any breathing difficulty during sleep. His usual sleep pattern was from 8 p.m. to 4 a.m. which was refreshing and deep. The patient did not report any other symptoms except for alcohol use. He started to consume alcohol at the age of 20 years. For the initial 5 years, he would consume 2 to 3 pegs of IMFL (whiskey) during night after meal, which increased to 2 quarters of IMFL (whiskey) after that. He would also consume a can of beer along with that. Whenever he would not consume alcohol he would have withdrawal symptoms and intense cravings. On some days, he would not be able to control his urge to drink and would consume almost $4-5$ quarters of IMFL in 2-3 hours. This pattern of use continued for the next 25 years till he was 50 years old. However, he was able to work properly, never had any complaints at workplace, his family had accepted his alcohol use as a norm so there was no family conflict as well. He reported to be abstinent from alcohol from age 50-55 without any apparent reason. At age 56 , he again began to consume alcohol, and for the last 11 years, he would consume it twice weekly during night with 2 pegs of IMFL at a time. There was no history of complicated alcohol withdrawal, features of liver dysfunction, and sudden loss of muscle tone or any other substance use. He did not report symptoms of any other medical disorder and neither had any surgery in past. His general physical examination revealed no abnormality in skin, hair, or otherwise. His vital parameters were within normal limits. In the mental status examination, his mood was euthymic, and higher mental functions were intact.

\section{Management and Outcome}

The patient was evaluated on the Epworth sleepiness scale for his daytime drowsiness and had a score of 18 (severe excessive daytime sleepiness). He was also advised certain investigations-CBC, RFT, LFT, vitamin B12, D3, thyroid profile, blood sugar, ECG, and MRI brain. All the investigations were within normal limits except for raised TSH. He was referred to the department of medicine for his hypothyroidism and was started on tab thyroxine $25 \mu \mathrm{g}$. After 2 weeks, he had minimal improvement in his symptoms and was concerned about it. He was then started on tab modafinil $50 \mathrm{mg}$ and reported improvement within a week.

\section{Discussion}

Narcolepsy is a rare, chronic nervous system disease that usually includes acute daytime drowsiness, episodic cataplexy, sleep paralysis, hypnagogic hallucination, and a disrupted sleep cycle. Narcolepsy was first reported and named by Gelineau in 1880 but the etiology remains unknown. It can be either hereditary (in 10-30\% of cases) or can occur following insults to the central nervous system including cerebral trauma, viral infection, immune dysfunction, brain tumors, multiple sclerosis, or severe emotional stress.

Chronic heavy alcohol use causes widespread damage to the central nervous system, inhibits the reticular activating system and thus precipitates episodes of narcolepsy and catalepsy. Our patients did not undergo any objective sleep analysis for their symptoms
The patient described in our first case had several of the cardinal symptoms of narcolepsy: (a) uncontrollable excessive daytime drowsiness, (b) cataplexy and (c) disrupted nocturnal sleep. These clinical characteristics are similar to those described in the literature and are listed as diagnostic criteria of narcolepsy in the 1997 International Classification of Sleep Disorders. ${ }^{3}$ The patient had a long history of drinking and was abstinent for only a month before presenting to us, so the insult to the central nervous system was ongoing. Besides alcohol dependence and narcolepsy he was also suffering from clinical depression.

Our second patient reported only excessive daytime drowsiness and responded to modafinil treatment. He was neither taking any medication nor reported insufficient sleep at the time of presentation.

Both of our patients did not undergo any objective sleep analysis for their symptoms which is a major drawback considering the diagnosis of narcolepsy. However, as we have ruled out other possible causes by clinical and diagnostic tests, alcohol-induced narcolepsy seems to be the most likely diagnosis. Still, the requirement of an objective sleep study for confirmation cannot be negated.

The treatment of narcolepsy is mainly symptomatic, aimed at suppressing symptoms and improving patients' quality of life. ${ }^{4}$ The most common modalities include stimulants and behavioral therapy to reduce acute daytime drowsiness, antidepressants to decrease episodes of cataplexy and other REM-related symptoms, and sedatives to improve the quality of nocturnal sleep..$^{5-7}$ In our case, the combination of the antidepressant sertraline (a selective serotonin reuptake inhibitor), modafinil (stimulant), and clonazepam (a nonbenzodiazepine hypnotic) controlled the symptoms.

\section{Conclusion}

Alcohol can cause a variety of sleep disorders including narcolepsy. Sleep-related disorders should be part of routine evaluation for alcohol use disorders.

\section{References}

1. Roehrs T, Roth T. Sleep, sleepiness, sleep disorders and alcohol use and abuse. Sleep Med Rev 2001;5(4):287-297. DOI: 10.1053/ smrv.2001.0162.

2. Barateau L, Jaussent I, Lopez R, et al. Smoking, alcohol, drug use, abuse and dependence in narcolepsy and idiopathic hypersomnia: a case-control study. Sleep 2016;39(3):573-580. DOI: 10.5665/ sleep. 5530.

3. Pan JY. Clinical diagnosis and therapy for sleep disorders. Guangzhou: South China University of Technology Press; 2001. p. 142-143 [in Chinese].

4. Zhang Y, Shen F. Progress and therapy for narcolepsy. J Med Res 2010;39(9):117-118 [in Chinese].

5. Dauvilliers Y, Arnulf I, Mignot E. Narcolepsy with cataplexy. Lancet 2007;369(9560):499-511. DOI: 10.1016/S0140-6736(07)60237-2.

6. Billiard M. Narcolepsy: current treatment options and future approaches. Neuropsychiatr Dis Treat 2008;4(3):557-566. PMID: 18830438.

7. Nishino S, Okuro M. Emerging treatments for narcolepsy and its related disorders. Expert Opin Emerg Drugs 2010;15(1):139-158. DOI: $10.1517 / 14728210903559852$. 